\title{
SEASONAL AND CYCLICAL LONG MEMORY
}

\author{
by \\ Josu Arteche \\ University of the Basque Country, Bilbao \\ and \\ Peter M Robinson \\ London School of Economics and Political Science
}

Contents:

Abstract

1. Introduction

2. Modelling Seasonality and Cycles

3. SCLM Processes

4. Estimation in SCLM Processes

5. Testing Seasonal/Cyclical Integration and Cointegration

6. Estimation of the Frequency $\mathrm{T}$

7. Conclusion and Extensions

References

List of previous papers

\begin{tabular}{ll} 
& The Suntory Centre \\
& Suntory and Toyota International Centres \\
& for Economics and Related Disciplines \\
& London School of Economics and Political \\
& Science \\
Hiscussion Paper & Houghton Street \\
No. EM/98/360 & London WC2A 2AE \\
September 1998 & Tel.: 0171-405 7686 \\
\hline
\end{tabular}

Research supported by ESRC grant R000235892. Josu Arteche also acknowledges financial support from the Bank of Spain and UPV grant 0.38.321-HB039/97. We thank the referees for their comments. 


\begin{abstract}
There has recently been great interest in time series with long memory, namely series whose dependence decays slowly in the sense that autocovariances are not summable and the spectral density is unbounded. This concept has been extended to SCLM (Seasonal/Cyclical Long Memory) where the dependence between seasonal or cyclic observations decays similarly slowly. We discuss issues related to SCLM processes such as modelling, estimation, statistical inference, applications and extensions.
\end{abstract}

Keywords: Long memory; seasonal time series; cyclic time series. JEL No.: C32

(C) by Josu Arteche and Peter M Robinson. All rights reserved. Short sections of text, not to exceed two paragraphs, may be quoted without explicit permission provided that full credit, including (c) notice, is given to the source. 


\section{INTRODUCTION}

Long memory of a covariance stationary series $x_{t}, t=0, \pm 1, \pm 2, \ldots$, may be modelled in the frequency domain by the spectral distribution function, $F(\lambda)$, or spectral density $f(\lambda)=$ $\mathrm{d} F(\lambda) / \mathrm{d} \lambda$, satisfying

$$
\gamma_{j}=\int_{-\pi}^{\pi} f(\lambda) \cos (j \lambda) \mathrm{d} \lambda
$$

where $\gamma_{j}=E\left(x_{t}-E x_{0}\right)\left(x_{t+j}-E x_{0}\right)$ is the lag-j autocovariance of $x_{t}$. In a semiparametric setup $f(\lambda)$ is typically assumed to behave as

$$
f(\lambda) \sim C|\lambda|^{-2 d} \quad \text { as } \lambda \rightarrow 0
$$

where $0<C<\infty$ and the memory or persistence parameter, $d$, satisfies $d<1 / 2$ for stationarity and $d>-1 / 2$ for invertibility. $x_{t}$ is said to have long memory if $d>0$, short memory if $d=0$ and negative memory if $d<0$. For reviews see Beran (1994a) or Robinson (1994c). Under additional assumptions (see Yong (1974))

$$
\gamma_{j} \sim K j^{2 d-1} \quad \text { as } j \rightarrow \infty,
$$

where $K$ is a positive constant when $0<d<1 / 2$. (??) implies that the autocovariances decay at a slow hyperbolic rate rather than the exponential one typical of stationary ARMA processes, and they are eventually positive.

Many time series move in a regular or quasi-regular manner showing a cyclical evolution that produces oscillating autocorrelations and peaks in the spectral density whose locations define the cycles, a spectral peak at frequency $\omega$ reflecting a cycle of period $2 \pi / \omega$. A particular case occurs when the spectral density has peaks at seasonal frequencies $\omega_{h}=2 \pi h / s, h=1,2, \ldots,[s / 2]$, where $s$ is the number of observations per year $(s=4$ for quarterly data, $s=12$ for monthly data) and $[s / 2]$ denotes the integer part of $s / 2$, that is $s / 2$ if $s$ is even and $(s-1) / 2$ if $s$ is odd. In this case we say that $x_{t}$ is a seasonal process. Nerlove (1964) described seasonality as "that characteristic of a time series that gives rise to spectral peaks at seasonal frequencies". In this sense we consider seasonality a special case of cyclical behaviour.

In this paper we focus on processes whose spectral density has a singularity or a zero at any frequency $\omega, 0<\omega \leq \pi$, such that

$$
f(\omega+\lambda) \sim C|\lambda|^{-2 d} \quad \text { as } \lambda \rightarrow 0,|d|<1 / 2
$$

where $C$ is a positive constant. Thus $f(\lambda)$ has a pole at $\lambda=\omega$ if $d>0$ and a zero if $d<0$. When $f(\lambda)$ satisfies (??) for every seasonal frequency $\omega=\omega_{h}, h=1,2, \ldots,[s / 2]$, possibly with the memory parameter, $d$, varying across $h$, we say that the process has "seasonal long memory". However, for non-seasonal time series, perhaps of annual data, we can have cyclic behaviour such that (??) holds for a single $\omega$ or for a single $\omega \in(0, \pi]$ as well as $\omega=0$. We thus use the terminology SCLM (Seasonal/Cyclical Long Memory) for processes satisfying (??) for one or more $\omega \in(0, \pi]$ (though strictly $-1 / 2<d<0$ entails "negative dependence", not long memory). 
SCLM processes might be described in terms of their autocovariances just as mentioned in (??) for standard long memory processes. A characteristic of autocovariances of SCLM processes is oscillating slow decay such that often $\gamma_{j}=O\left(j^{2 d-1}\right)$ as $j \rightarrow \infty$ but with oscillations whose amplitude depends on $\omega$ instead of the eventual monotonic decay in (??) of standard long memory processes at frequency zero .

The models traditionally used for seasonal and cyclical time series are stationary short memory processes on the one hand, or nonstationary processes due to a deterministic component such as seasonal dummies or to a stochastic trend such as seasonal unit roots. This work is reviewed in Section 2 in order to place SCLM in some perspective. The modelling of SCLM is described in more detail in Section 3. Section 4 discusses several parametric and semiparametric methods of estimation in SCLM processes. Tests of seasonal integration and cointegration are reviewed in Section 5. All this work assumes knowledge of the location of the poles/zeros in $f(\lambda)$, as is reasonable in a seasonal setting, but not necessary in a cyclic one. Section 6 describes approaches for estimating $\omega$ in parametric and semiparametric SCLM processes. Section 7 concludes the paper with some mention of extensions and applications.

\section{MODELLING SEASONALITY AND CYCLES}

Seasonality has traditionally been considered a nuisance that obscure the more important components of time series (e.g. growth and cyclical components), and several seasonal adjustment procedures have been proposed. They are typically based on the idea that a time series, possibly after logarithmic transformation, is additively composed of three different components, the trend-cycle, $T_{t}$, the seasonal, $S_{t}$, and the irregular component, $I_{t}$,

$$
x_{t}=T_{t}+S_{t}+I_{t}
$$

Traditionally $T_{t}$ includes also the possibility of a cyclical component, considering the cycle as a periodic component with period larger than the number of observations per year. This implies a spectral peak at some frequency between zero and $2 \pi / s$ which may be indistinguishable from a stochastic trend, characterized by a spectral pole at the origin. However, there may be cycles of period different from the seasonal ones, $s / j$, for $j=1,2, \ldots,[s / 2]$. To allow for this behaviour we can include a cyclic component, $C_{t}$, in (??),

$$
x_{t}=T_{t}+C_{t}+S_{t}+I_{t}
$$

The additive form in (??) and (??) is often known as Unobserved Component (UC) or Structural Time Series model. The seasonally adjusted series is obtained by subtracting an estimate of $S_{t}$. We group the different methods of estimation of $S_{t}$ and adjustment of $x_{t}$ in two classes, "modelfree" and "model-based" adjusting procedures. The "model-free" techniques ignore the seasonal and other structure of the series. They are based on the application of a succession of moving averages, perhaps the most widely used being the US Bureau of the Census X-11 procedure 
(Shiskin et al. (1967)) and the X-11 ARIMA (Dagum (1980)) which apply two-sided filters. The "model-based" seasonal adjustment procedures adapt to the characteristics of each series by estimation of parametric models. Some of these models are described below.

Seasonal adjustment procedures have been criticized for causing undesirable effects such as spectral dips at seasonal frequencies or distortion of the spectral density at other frequencies (see Nerlove (1964) or Bell and Hillmer (1984)). Furthermore, the UC models in (??) and (??) suppose that each component in $x_{t}$ can be specified separately and independently of the remainder, whereas the same model can include two or more components (for example the stochastic seasonal processes classified as b), c) and d) below include an irregular component). Such factors have encouraged the use of seasonally unadjusted data.

Most of the processes described in this section are seasonal, modelling a specific cyclical behaviour. However, other cyclic patterns can be modelled similarly by suitably choosing the dummy variables, cosinusoids or lag operators in the models described below.

One of the earliest models for seasonality is the deterministic, strictly periodic form

$$
x_{t}=\sum_{k=1}^{s} a_{k} D_{k t}
$$

where $D_{k t}=1$ if $t-k$ is a multiple of $s$ (the number of observations per year) and 0 otherwise and

$$
\sum_{k=1}^{s} a_{k}=0
$$

which may be achieved by subtracting a constant from the original series. We can rewrite (??) as a function of sine and cosine waves,

$$
x_{t}=\sum_{h=1}^{\left[\frac{s}{2}\right]} \Psi_{h, t},
$$

where

$$
\begin{aligned}
\Psi_{h, t} & =\alpha_{h} \cos \left(\omega_{h} t\right)+\beta_{h} \sin \left(\omega_{h} t\right), \omega_{h}=\frac{2 \pi h}{s}, \\
\alpha_{h} & =\frac{2}{s} \sum_{k=1}^{s} a_{k} \cos \left(k \omega_{h}\right), \\
\beta_{h} & =\frac{2}{s} \sum_{k=1}^{s} a_{k} \sin \left(k \omega_{h}\right),
\end{aligned}
$$

for $1 \leq h<s / 2$, and if $s$ is even $\beta \frac{s}{2} \sin \left(k \omega_{\frac{s}{2}}\right)$ is zero and

$$
\alpha_{\frac{s}{2}}=\frac{1}{s} \sum_{k=1}^{s} a_{k} \cos \left(k \omega_{\frac{s}{2}}\right)
$$

(see Hannan (1963)). $x_{t}$ in (??) can equivalently be written $x_{t}=\sum_{h=1}^{[s / 2]} r_{h} \cos \left(\omega_{h} t-\theta_{h}\right)$ where $r_{h}=\sqrt{\alpha_{h}^{2}+\beta_{h}^{2}}$ is the $h$-th amplitude and $\theta_{h}=\arctan \left(\beta_{h} / \alpha_{h}\right)$ is the $h$-th phase. It is rarely plausible that time series have such a rigid deterministic behaviour as (??) or (??) impose, so 
a stochastic error term is often added. If this irregular component is well behaved and the frequencies $\omega_{h}$ are known, then $\alpha_{h}$ and $\beta_{h}$ in (??) or $a_{k}$ in (??) can be estimated through simple regression methods. In fact least squares estimates have desirable orthogonality properties under uncorrelated errors, and are Gauss-Markov efficient under quite general (albeit short memory) autocorrelated errors.

The processes (??) and (??) are completely deterministic, and if $\alpha_{h}, \beta_{h}$ are fixed parameters they are non-stationary so that it does not make sense to speak of a spectral distribution function or spectral density. However the spectral behaviour of stochastic seasonal time series will give us relevant information on the characteristics of the process. According to spectral characteristics we distinguish four classes of stochastic seasonal/cyclical processes:

a) Stationary with spectral distribution function with jumps and thus not absolutely continuous.

b) Stationary with absolutely continuous spectral distribution function everywhere and smooth, positive, spectral density.

c) Stationary with absolutely continuous spectral distribution function but spectral density with one or more singularities or zeros.

d) Non-stationary so that no spectral distribution function exists.

a) Stationary process with jumping spectral distribution. This kind of process is defined by (??) and (??) but $\Psi_{h, t}$ is made stochastic by allowing $\alpha_{h}$ and $\beta_{h}$ to be random variables satisfying

$$
\begin{aligned}
& E\left[\alpha_{h}\right]=E\left[\beta_{h}\right]=0, E\left[\alpha_{h}^{2}\right]=E\left[\beta_{h}^{2}\right]=\sigma_{h}^{2} \text { for all } h \\
& E\left[\alpha_{h} \alpha_{i}\right]=E\left[\beta_{h} \beta_{i}\right]=0 h \neq i, E\left[\alpha_{h} \beta_{i}\right]=0 \text { for all } h, i .
\end{aligned}
$$

Under (??), $x_{t}$ is covariance stationary with lag- $j$ autocovariance

$$
\gamma_{j}=E\left(x_{t} x_{t-j}\right)=\sum_{h=1}^{\left[\frac{s}{2}\right]} \sigma_{h}^{2} \cos \left(\omega_{h} j\right)=\int_{-\pi}^{\pi} \cos (j \lambda) \mathrm{d} F(\lambda) \quad j=0, \pm 1 \ldots .
$$

Although $\alpha_{h}$ and $\beta_{h}$ are random variables, they are fixed in a particular realization. Thus, although $\Psi_{h, t}$ is stationary, the model is still deterministic, only two observations are necessary to determine $\alpha_{h}$ and $\beta_{h}$, and once this has been done the remainder of the series can be forecast with zero mean squared error. The spectral distribution function, $F(\lambda)$, is a step function consisting of jumps of magnitude $\sigma_{h}^{2} / 2$ at frequencies $-\omega_{h}$ and $\omega_{h}$, for $h=1, \ldots,[s / 2]$. Since $F(\lambda)$ is not continuous the spectral density does not exist. However, in a similar manner as Stieltjes integration is carried out, we can define the so-called line or discrete spectrum, that is a discrete function with values $\sigma_{h}^{2} / 2$ at frequencies $-\omega_{h}$ and $\omega_{h}$ for $h=1, \ldots,[s / 2]$. The line spectrum at $\omega_{h}$ gives the relative importance of a cycle of period $s / h$ in the variance of $x_{t}$.

b) Stationary process with absolutely continuous spectral distribution and smooth spectral density. The models in (??) and (??) assume that the cyclic behaviour in $x_{t}$ is constant across time and does not change its form. However, in many time series the seasonal/cyclical behaviour 
is likely to change across time. Of course the variation must be slow (otherwise we cannot speak of seasonality or cycle) in such a way that the periodical structure seems to persist and the series has a quasi-periodic behaviour. Hannan (1964) allows for this behaviour in the model

$$
x_{t}=\sum_{h=1}^{\left[\frac{s}{2}\right]} \Psi_{h, t}, \quad \Psi_{h, t}=\alpha_{h, t} \cos \left(\omega_{h} t\right)+\beta_{h, t} \sin \left(\omega_{h} t\right),
$$

where $\omega_{h}=2 \pi h / s$ are seasonal frequencies and $\alpha_{h, t}$ and $\beta_{h, t}$ are not constant but evolving with time. Hannan (1964) assumed

$$
\begin{aligned}
& E\left[\alpha_{h, t}\right]=E\left[\beta_{h, t}\right]=0 \text { for all } h \text { and all } t \\
& E\left[\alpha_{h, t} \alpha_{h, t-j}\right]=E\left[\beta_{h, t} \beta_{h, t-j}\right]=c_{h} \rho_{h}^{j} \\
& E\left[\alpha_{h, t} \alpha_{i, s}\right]=E\left[\beta_{h, t} \beta_{i, s}\right]=0 \text { for } h \neq i \text { and all } t, s \\
& E\left[\alpha_{h, t} \beta_{i, s}\right]=0 \text { for all } h, i \text { and all } t, s .
\end{aligned}
$$

Thus the lag- $j$ autocovariance of $\Psi_{h, t}$ is

$$
E\left[\Psi_{h, t} \Psi_{h, t-j}\right]=c_{h} \rho_{h}^{j} \cos \left(\omega_{h} j\right) .
$$

Stationarity of $\Psi_{h, t}$ entails $\left|\rho_{h}\right|<1$. However, $\rho_{h}$ has to be close to 1 to avoid a fast changing behaviour of $\Psi_{h, t}$. When $\left|\rho_{h}\right|<1, \Psi_{h, t}$ is stationary and non deterministic with absolutely continuous spectral distribution and smooth spectral density,

$$
\begin{aligned}
f_{h}(\lambda) & =\frac{c_{h}}{2 \pi} \sum_{j=-\infty}^{\infty} \rho_{h}^{j} \cos \left(\omega_{h} j\right) \cos (\lambda j) \\
& =\frac{c_{h}}{4 \pi}\left\{\frac{1-\rho_{h}^{2}}{1+\rho_{h}^{2}-2 \rho_{h} \cos \left(\lambda-\omega_{h}\right)}+\frac{1-\rho_{h}^{2}}{1+\rho_{h}^{2}-2 \rho_{h} \cos \left(\lambda+\omega_{h}\right)}\right\}
\end{aligned}
$$

which, for $\rho_{h}$ near to unity, will concentrate around $\lambda=\omega_{h}$. Hannan et al. (1970) considered a parameterization of $\alpha_{h, t}$ and $\beta_{h, t}$ obeying (??),

$$
\alpha_{h, t}=\rho_{h} \alpha_{h, t-1}+\varepsilon_{h, t}, \quad \beta_{h, t}=\rho_{h} \beta_{h, t-1}+\varepsilon_{h, t}^{\dagger}, \quad\left|\rho_{h}\right|<1,
$$

where $\varepsilon_{h, t}$ and $\varepsilon_{h, t}^{\dagger}$ have zero mean and common variance $\sigma_{h}^{2}$, and all correlations between $\varepsilon, \varepsilon^{\dagger}$ and between two time points and for differing values of $h$ vanish. Substituting (??) in $\Psi_{h, t}$ in (??), we find that $\Psi_{h, t}$ is an $\operatorname{ARMA}(2,1)$ process

$$
\left(1-2 \rho_{h} \cos \left(\omega_{h}\right) L+\rho_{h}^{2} L^{2}\right) \Psi_{h, t}=\eta_{h, t}-\rho_{h} \cos \left(\omega_{h}\right) \eta_{h, t-1}-\rho_{h} \sin \left(\omega_{h}\right) \eta_{h, t-1}^{\dagger},
$$

where

$$
\begin{aligned}
\eta_{h, t} & =\varepsilon_{h, t} \cos \left(\omega_{h} t\right)+\varepsilon_{h, t}^{\dagger} \sin \left(\omega_{h} t\right) \\
\eta_{h, t}^{\dagger} & =\varepsilon_{h, t} \sin \left(\omega_{h} t\right)-\varepsilon_{h, t}^{\dagger} \cos \left(\omega_{h} t\right)
\end{aligned}
$$

are thus zero mean random variables with variance $\sigma_{h}^{2}$ and inherit the uncorrelatedness properties of $\varepsilon_{h, t}$ and $\varepsilon_{h, t}^{\dagger}$. The lag- $j$ autocovariance and spectral density of $\Psi_{h, t}$ are (??) and (??) with $c_{h}=\sigma_{h}^{2} /\left(1-\rho_{h}^{2}\right)$. Consequently the spectrum of $x_{t}$ is a smooth function

$$
f(\lambda)=\sum_{h=1}^{\left[\frac{s}{2}\right]} f_{h}(\lambda)
$$


which shows peaks (the sharper the closer $\rho_{h}$ is to 1) around seasonal frequencies $\omega_{h}, h=$ $1,2, \ldots,[s / 2]$.

In addition to the specific ARMA in (??) we can use many other ARMA processes to model a changing cyclical behaviour. In particular, if the spectrum of an $\operatorname{AR}(2),\left(1-\phi_{1} L-\phi_{2} L^{2}\right) x_{t}=\varepsilon_{t}$, contains a peak at frequency $\lambda^{*}$ within the range $0<\lambda^{*}<\pi$, its exact position is

$$
\lambda^{*}=\cos ^{-1}\left[\frac{-\phi_{1}\left(1-\phi_{2}\right)}{4 \phi_{2}}\right] .
$$

For example the spectrum of the AR part in (??) has a peak at

$$
\lambda^{*}=\cos ^{-1}\left[\frac{\left(1+\rho_{h}^{2}\right) \cos \omega_{h}}{2 \rho_{h}}\right]
$$

so that $\lambda^{*}$ is closer to $\omega_{h}$ the closer $\rho_{h}$ is to 1 . We can also use the seasonal lag operator, $L^{s}$, $\left(L^{s} x_{t}=x_{t-s}\right)$ to define the seasonal ARMA $(1,1)$ model

$$
\left(1-\phi_{s} L^{s}\right) x_{t}=\left(1+\theta_{s} L^{s}\right) \varepsilon_{t}
$$

where $\varepsilon_{t}$ is white noise with variance $\sigma^{2}$. When $\phi_{s}$ and $\theta_{s}$ are inside the unit circle, $x_{t}$ is stationary and invertible with smooth spectral density

$$
f(\lambda)=\frac{\sigma^{2}}{2 \pi} \frac{1+\theta_{s}^{2}+2 \theta_{s} \cos (\lambda s)}{1+\phi_{s}^{2}-2 \phi_{s} \cos (\lambda s)} .
$$

If $\phi_{s}>0$ and $\theta_{s}>0, f(\lambda)$ exhibits peaks at the seasonal harmonic frequencies, $\omega_{h}=2 \pi h / s$, $h=1,2, \ldots,[s / 2]$, as well as at zero. More general seasonal ARMA processes can be defined as

$$
\Phi_{s}\left(L^{s}\right) x_{t}=\Theta_{s}\left(L^{s}\right) \varepsilon_{t}
$$

where $\Phi_{s}\left(L^{s}\right)$ and $\Theta_{s}\left(L^{s}\right)$ are polynomials in the seasonal lag operator with zeros outside the unit circle (see Box and Jenkins (1976)).

c) Stationary process with absolutely continuous spectral distribution and singularities or zeros in its spectral density. The structure of $\alpha_{h}$ and $\beta_{h}$ in (??) may generate a relatively rapid change in the seasonal pattern, whereas the definition of seasonality implies a regular or quasiregular behaviour. The closer $\rho_{h}$ is to 1 the more regular the movement of $\Psi_{h, t}$. In fact we can choose $\rho_{h}=1$, but in this case $\Psi_{h, t}$ ceases to be stationary. Instead we can assume that $\alpha_{h, t}$ and $\beta_{h, t}$ evolve as

$$
(1-L)^{d_{h}} \alpha_{h, t}=\varepsilon_{h, t} \quad, \quad(1-L)^{d_{h}} \beta_{h, t}=\varepsilon_{h, t}^{\dagger},
$$

where $\varepsilon_{h, t}$ and $\varepsilon_{h, t}^{\dagger}$ are defined as in (??). Thus $\alpha_{h, t}$ and $\beta_{h, t}$ are fractional $\operatorname{ARIMA}\left(0, d_{h}, 0\right)$ processes and they are stationary if $d_{h}<1 / 2$ and invertible if $d_{h}>-1 / 2$ (see Hosking (1981)). The slowly changing behaviour necessary for seasonality requires $d_{h}>0$ and stationarity entails $d_{h}<1 / 2$. Under these circumstances the spectral density of $\alpha_{h, t}$ and $\beta_{h, t}, f_{0}(\lambda)$, satisfies (??) for $\omega=0$. Their lag- $j$ autocovariance is

$$
\gamma_{h, j}^{\dagger}=E\left[\alpha_{h, t} \alpha_{h, t-j}\right]=E\left[\beta_{h, t} \beta_{h, t-j}\right]=\sigma_{h}^{2} \frac{\Gamma\left(1-2 d_{h}\right) \Gamma\left(j+d_{h}\right)}{\Gamma\left(d_{h}\right) \Gamma\left(1-d_{h}\right) \Gamma\left(j+1-d_{h}\right)} .
$$


Thus the lag- $j$ autocovariance of $\Psi_{h, t}$ is

$$
E\left[\Psi_{h, t} \Psi_{h, t-j}\right]=\gamma_{h, j}^{\dagger} \cos \left(j \omega_{h}\right)
$$

and its spectral density is

$$
\begin{aligned}
f_{h}(\lambda) & =\frac{1}{2 \pi} \sum_{j=-\infty}^{\infty} \gamma_{h, j}^{\dagger} \cos \left(j \omega_{h}\right) e^{-i \lambda j}=\frac{1}{4 \pi} \sum_{j=-\infty}^{\infty} \gamma_{h, j}^{\dagger} e^{-i \lambda j}\left(e^{i j \omega_{h}}+e^{-i j \omega_{h}}\right) \\
& =\frac{1}{2} f_{0}\left(\lambda-\omega_{h}\right)+\frac{1}{2} f_{0}\left(\lambda+\omega_{h}\right) .
\end{aligned}
$$

The multiplication of $\alpha_{h, t}$ by $\cos \left(\omega_{h} t\right)$ and $\beta_{h, t}$ by $\sin \left(\omega_{h} t\right)$ produces a phase shift such that the spectral pole moves from zero in $\alpha_{h, t}$ and $\beta_{h, t}$ to $\omega_{h}$ in $\Psi_{h, t}$. Thus, the process defined by equations (??) and (??) has an absolutely continuous spectral distribution but its spectral density is not smooth, but goes to $\infty$ (if $d_{h}>0$ ) or is zero (if $d_{h}<0$ ), at frequencies $\pm \omega_{h}$ as described in (??). This is the SCLM property that characterizes the processes we focus attention on in this paper. A more detailed description of models with this property is given in next section.

d) Non-stationary and non-deterministic stochastic seasonal process. If $\alpha_{h, t}$ and $\beta_{h, t}$ are determined by the fractional ARIMAs in (??) but with $d_{h} \geq 1 / 2$, then they, and thus $\Psi_{h, t}$ in (??), are non-stationary. In this case there does not exist a spectral distribution. Nevertheless, the frequency domain is still an adequate framework to detect seasonality using the pseudospectrum. If $u_{t}=\tau(L) x_{t}$ is stationary with spectrum $f_{u}(\lambda)$, the pseudospectrum of $x_{t}$ is $f(\lambda)=\left|\tau\left(e^{i \lambda}\right)\right|^{-2} f_{u}(\lambda)$. For example, if $d_{h}=1$ in (??) or equivalently $\rho_{h}=1$ in (??), then $\Psi_{h, t}$ is a non-stationary $\operatorname{ARMA}(2,1)$ process

$$
\tau_{h}(L) \Psi_{h, t}=\eta_{h, t}-\cos \left(\omega_{h}\right) \eta_{h, t-1}-\sin \left(\omega_{h}\right) \eta_{h, t-1}^{\dagger}
$$

where $\tau_{h}(L)=1-2 \cos \left(\omega_{h}\right) L+L^{2}$. The non-stationarity comes from the fact that the AR polynomial, $\tau_{h}(L)$, has zeros at $\cos \omega_{h} \pm \sqrt{\cos ^{2} \omega_{h}-1}$, with modulus one. However $\tau_{h}(L) \Psi_{h, t}$ is a stationary $\operatorname{MA}(1)$. Since $\left|\tau_{h}\left(e^{i \lambda}\right)\right|^{-2}=\left(2\left(\cos \omega_{h}-\cos \lambda\right)\right)^{-2}$ diverges at $\lambda= \pm \omega_{h}$, then the pseudospectrum of $\Psi_{h, t}$ goes to infinity at frequencies $\pm \omega_{h}$, reflecting a strong cyclical pattern with period $2 \pi / \omega_{h}=s / h$. Hannan et al. (1970) estimated this model using optimal signal extraction methods (see also Hannan (1967)).

In the Box-Jenkins framework we can define the seasonal ARIMA(P,D,Q) time series

$$
\Phi_{s}\left(L^{s}\right)\left(1-L^{s}\right)^{D} x_{t}=\Theta_{s}\left(L^{s}\right) \varepsilon_{t}
$$

where the $\varepsilon_{t}$ are white noise $\left(0, \sigma^{2}\right), \Phi_{s}\left(L^{s}\right)$ and $\Theta_{s}\left(L^{s}\right)$ are polynomials in the lag operator with zeros outside the unit circle, and $D$ is a positive integer in Box and Jenkins (1976) but could instead be fractional (Hosking (1984)). Then (??) defines the fractional seasonal ARIMA(P,D,Q), that is stationary if $D<1 / 2$ and non-stationary if $D \geq 1 / 2$. The spectrum $(D<1 / 2)$ or pseudospectrum (if $D \geq 1 / 2)$ of $x_{t}$ is

$$
f(\lambda)=\frac{\sigma^{2}}{2 \pi} \frac{\left|\Theta_{s}\left(e^{i \lambda s}\right)\right|^{2}}{\left|\Phi_{s}\left(e^{i \lambda s}\right)\right|^{2}}\left(2 \sin \frac{\lambda s}{2}\right)^{-2 D}
$$


and diverges if $D>0$ or is zero if $D<0$ at frequencies $\omega_{h}=2 \pi h / s, h=0, \ldots,[s / 2]$, that is at the origin and seasonal frequencies. The seasonal difference operator, $\left(1-L^{s}\right)$, can be written as the product of the difference operator, $(1-L)$, and the seasonal summation operator, $S(L)=\left(1+L+\ldots+L^{s-1}\right)$, such that the pole in (??) at the origin corresponds to the operator $(1-L)$, and the spectral poles at seasonal frequencies are due to $S(L)$. Thus $\left(1-L^{s}\right)$ includes a stochastic trend in addition to the seasonal factor. This is why sometimes (e.g. Harvey (1989)), $S(L)$ is used instead of $\left(1-L^{s}\right)$ to model the seasonal component of the UC models in (??) and (??).

Another type of non-stationarity may be described by a different data generating process for each season. This phenomenon is often modelled via the Periodic ARIMA process (e.g. Troutman (1979), Tiao and Grupe (1980), Osborn (1991), Franses and Ooms (1995)),

$$
\Phi_{q}(L)\left(1-L^{s}\right)^{d_{q}} x_{T}^{q}=\Theta_{q}(L) \varepsilon_{T}^{q} \quad q=1, \ldots, s, \quad T=1,2, \ldots,
$$

where $\varepsilon_{T}^{q}$ is white noise with variance $\sigma_{q}^{2}$, the index $q$ indicates the season or situation of the observation in the cycle (for example different months) and $T$ represents the year such that $x_{T}^{q}=x_{(T-1) s+q}$. Thus, (??) allows for $s$ different models, one per season. When the zeros of $\Phi_{q}(L)$ and $\Theta_{q}(L)$ lie outside the unit circle, and $d_{q}<1 / 2$, then (??) is stationary for every $q=1,2, \ldots, s$. Although $x_{t}^{q}$ may be stationary, $x_{t}$ is non-stationary if some parameters vary with $q$. In this case the autocovariances of $x_{t}$ depend on $q$ and therefore are not time invariant and we cannot use frequency domain techniques. This kind of process is usually analysed in a multivariate set-up using a vector ARMA representation. Define the $s \times 1$ vector $z_{T}=$ $\left(x_{T}^{1}, \ldots, x_{T}^{s}\right)^{\prime}=\left(x_{(T-1) s+1}, \ldots, x_{T s}\right)^{\prime}$. The periodic process in (??) can be written in vector ARMA form as

$$
A\left(L^{*}\right) C\left(L^{*}\right) z_{T}=B\left(L^{*}\right) u_{T} \quad T=1,2, \ldots
$$

where $u_{T}=\left(\varepsilon_{T}^{1}, \ldots, \varepsilon_{T}^{q}\right)^{\prime}, C\left(L^{*}\right)=\operatorname{diag}\left\{\left(1-L^{*}\right)^{d_{q}}\right\}, A\left(L^{*}\right)$ and $B\left(L^{*}\right)$ are matrix polynomials in $L^{*}$, and the operator $L^{*}$ is the lag operator for the index $T, L^{*} z_{T}=z_{T-1}$. This implies seasonal difference in the elements of $z_{T}, L^{*} x_{T}^{q}=L^{s} x_{(T-1) s+q}=x_{(T-2) s+q}=x_{T-1}^{q}$. The vector $z_{T}$ is stationary if $d_{q}<1 / 2$ for $q=1, \ldots, s$, and $|A(z)|$ has zeros outside the unit circle, and is invertible if $d_{q}>-1 / 2$ for $q=1, \ldots, s$, whereas the zeros of $|B(z)|$ lie outside the unit circle. Under stationarity $z_{T}$ has a spectral density matrix $f_{z}(\lambda)$. Although $x_{t}$ is non-stationary the expectation of the sample autocovariances of $x_{t}$ converges to the autocovariances of a stationary process with spectral density function

$$
f(\lambda)=\frac{1}{s} R\left(e^{i \lambda}\right)^{\prime} f_{z}(s \lambda) R\left(e^{-i \lambda}\right)
$$

where $R(r)$ is a $s \times 1$ vector with $k$-th element $r^{k}$ (Tiao and Grupe (1980)). Thus asymptotically we can use (??) to classify periodic processes in the same way as non-periodic seasonal models. 


\section{SCLM PROCESSES}

This section describes commonly used parametric models of the class c) of stochastic seasonal processes introduced in the previous section, that is processes whose spectral density satisfies (??). We say that such processes have SCLM, and using the notation in Engle et al. (1989) we denote them by $I_{\omega}(d)$ (integrated of order $d$ at $\omega$ ).

Though (??) is a semiparametric condition, only imposing knowledge of $f(\lambda)$ around $\omega$, it is interesting to describe parametric processes satisfying (??), specifying short memory as well as long memory components of $x_{t}$, for example for the purpose of Monte Carlo simulation. Some examples have been introduced in the previous section (e.g. (??) and (??) or (??)). In case of Gaussianity it suffices to specify the mean, $\mu$, and $f(\lambda)$ for all $\lambda \in(-\pi, \pi]$, or equivalently $\gamma_{j}$, for all $j$. Autocovariances of SCLM processes have a slow decay typical of long memory along with oscillations depending on the frequency $\omega$ such that, for $d>0, \sum\left|\gamma_{j}\right|=\infty$, although this is consistent with $\sum \gamma_{j}$, and thus $f(0)$, being finite. These observations apply to non-Gaussian (finite variance) series as well as Gaussian ones, though there remains the possibility that $x_{t}$ may not exhibit long memory in second moments but in some other way (for example $x_{t}^{2}$ could have long memory), as briefly discussed in Section 7 .

Two SCLM models have been stressed in the literature, being natural extensions of models for long memory at zero frequency, namely the fractional noise and the fractional ARIMA.

\subsection{Seasonal Fractional Noise}

This kind of stationary process is characterized by a spectral density

$$
f(\lambda)=c|1-\cos (s \lambda)| \sum_{j=-\infty}^{\infty}\left|\lambda+\frac{2 \pi}{s} j\right|^{-2(1+d)}
$$

and lag- $j$ autocovariance

$$
\gamma_{j}=\frac{V\left(x_{1}\right)}{2}\left(\left|\frac{j}{s}+1\right|^{2 d+1}-2\left|\frac{j}{s}\right|^{2 d+1}+\left|\frac{j}{s}-1\right|^{2 d+1}\right)
$$

where $s$ is the number of observations per year, $c$ is a positive constant and $d<1 / 2$ (see Jonas (1983), Carlin and Dempster (1989) or Ooms (1995)). The spectrum in (??) satisfies (??) for $\omega=2 \pi h / s, h=0,1, \ldots,[s / 2]$, the $\gamma_{j}$ in (??) have slow and oscillating decay as $j \rightarrow \infty$, and if $d>0$ they are not absolutely summable. This kind of process generalizes the fractional noise described by Mandelbrot and Van Ness (1968), characterized by (??) or (??) with $s=1$, and having typical long memory behaviour at frequency zero.

\subsection{SCLM in the Box-Jenkins set-up}

Andel (1986), and later and in more depth Gray et al. (1989,1994), analysed the so-called Gegenbauer process

$$
\left(1-2 L \cos \omega+L^{2}\right)^{d} x_{t}=u_{t}
$$


where $u_{t}$ has positive and continuous spectrum, $f_{u}(\lambda)$, and $d$ can be any real number. For example when $u_{t}$ is a stationary and invertible $\operatorname{ARMA}(\mathrm{p}, \mathrm{q})(? ?)$ is called GARMA (Gegenbauer ARMA). The spectral density of $x_{t}$ in (??) is

$$
f(\lambda)=(2(\cos \omega-\cos \lambda))^{-2 d} f_{u}(\lambda)
$$

and satisfies (??), so $x_{t}$ has SCLM at frequency $\omega$ for $|d|<1 / 2$ when $\omega \neq 0, \pi$. When $\omega=0$ and $u_{t}$ is an $\operatorname{ARM} A(p, q)$, then (??) is the fractional $\operatorname{ARIMA}(p, 2 d, q),(1-L)^{2 d} x_{t}=u_{t}$, so that $x_{t}$ is stationary if $d<1 / 4$ and invertible when $d>-1 / 4$. If $\omega=\pi, x_{t}$ is stationary if $d<1 / 4$ and invertible when $d>-1 / 4$. When $u_{t}$ is $i i d\left(0, \sigma^{2}\right)$, and $d<1 / 2$, the autocovariances of $x_{t}$ are

$$
\gamma_{j}=\frac{\sigma^{2}}{2 \sqrt{\pi}} \Gamma(1-2 d)(2 \sin \omega)^{\frac{1}{2}-2 d}\left[P_{j-\frac{1}{2}}^{2 d-\frac{1}{2}}(\cos \omega)+(-1)^{j} P_{j-\frac{1}{2}}^{2 d-\frac{1}{2}}(-\cos \omega)\right]
$$

where $P_{a}^{b}(z)$ are associated Legendre functions (Chung(1996a)). The asymptotic behaviour of $\gamma_{j}$ in (??) is

$$
\gamma_{j} \sim K \cos (j \omega) j^{2 d-1} \quad \text { as } j \rightarrow \infty
$$

where $K$ is a finite constant that depends on $d$ but not on $j$ (see Gray et al. (1989) or Chung (1996a)), so $\gamma_{j}$ has the slow and oscillating decay typical of SCLM.

Hosking (1984), Porter-Hudak (1990) and Ray (1993) among others, proposed use of the fractional seasonal difference operator, $\left(1-L^{s}\right)^{d}$, where $d$ can be any real number. Porter-Hudak (1990) used the operator $\left(1-L^{12}\right)^{d}$ in monthly monetary USA aggregates and Ray (1993) used $\left(1-L^{3}\right)^{d_{3}}\left(1-L^{12}\right)^{d_{12}}$ for monthly IBM revenue data. Note that for even $s,\left(1-L^{s}\right)^{d}$ can be decomposed into the product of operators of type $\left(1-2 L \cos \omega+L^{2}\right)^{d}$. For instance if $s=4$,

$$
\left(1-L^{4}\right)^{d}=\left(1-2 L \cos \omega_{0}+L^{2}\right)^{\frac{d}{2}}\left(1-2 L \cos \omega_{1}+L^{2}\right)^{d}\left(1-2 L \cos \omega_{2}+L^{2}\right)^{\frac{d}{2}}
$$

for $\omega_{0}=0, \omega_{1}=\pi / 2$ and $\omega_{2}=\pi$. Thus $x_{t}$ in $\left(1-L^{4}\right)^{d} x_{t}=u_{t}$ is $I_{0}(d), I_{\frac{\pi}{2}}(d)$ and $I_{\pi}(d)$.

In order to allow for different persistence parameters across different frequencies, Chan and Terrin (1995), Chan and Wei (1988), Giraitis and Leipus (1995) and Robinson (1994a) used the model

$$
(1-L)^{d_{0}}\left\{\prod_{j=1}^{h-1}\left(1-2 L \cos \omega_{j}+L^{2}\right)^{d_{j}}\right\}(1+L)^{d_{h}} x_{t}=u_{t}
$$

where the $\omega_{j}$ can be any frequencies between 0 and $\pi$ and $u_{t}$ has continuous and positive spectrum. Thus $x_{t}$ in (??) is $I_{\omega_{j}}\left(d_{j}\right)$ for $j=0,1,2, \ldots, h$, where $\omega_{0}=0$ and $\omega_{h}=\pi$. When $u_{t}$ is a stationary and invertible ARMA, Giraitis and Leipus (1995) used the terminology ARUMA for such $x_{t}$. When $\left|d_{j}\right|<1 / 2$ for $j=0,1, \ldots, h,($ ??) can be expressed as

$$
\sum_{j=0}^{\infty} \pi_{j} x_{t-j}=u_{t}
$$

or

$$
x_{t}=\sum_{j=0}^{\infty} \psi_{j} u_{t-j}
$$


where $\pi_{0}=\psi_{0}=1$ and

$$
\pi_{j}=\sum_{\substack{0 \leq k_{0}, \ldots, k_{h} \leq j \\ k_{0}+\ldots+k_{h}=j}} C_{k_{0}}^{\left(-d_{0} / 2\right)}\left(\eta_{0}\right) C_{k_{1}}^{\left(-d_{1}\right)}\left(\eta_{1}\right) \ldots C_{k_{h-1}}^{\left(-d_{h-1}\right)}\left(\eta_{h-1}\right) C_{k_{h}}^{\left(-d_{h} / 2\right)}\left(\eta_{h}\right)
$$

for $j=1, \ldots$, where $\eta_{i}=\cos \omega_{i}, i=0,1, \ldots, h$, and $C_{k}^{(d)}(x)$ are orthogonal Gegenbauer polynomials. Similarly $\psi_{j}$ is (??) with $d_{0}, \ldots, d_{h}$ instead of $-d_{0}, \ldots,-d_{h}$ (see Giraitis and Leipus (1995)). The weights $\pi_{j}$ in (??) have the asymptotic behaviour

$$
\pi_{j} \sim K\left[j^{-1-d_{0}}+(-1)^{j} j^{-1-d_{h}}+\sum_{k=1}^{h-1} j^{-d_{k}-1}\left(\cos \left(\omega_{k} j\right)+v_{k}\right)\right]
$$

where $K$ is a finite constant and $v_{k}$ is a constant depending on $d_{0}, \ldots, d_{h}$ and $\omega_{k}$. Similarly the $\psi_{j}$ behave asymptotically as (??) with $d_{0}, \ldots, d_{h}$ instead of $-d_{0}, \ldots,-d_{h}$.

The complicated form of (??) impedes calculation of explicit formulae for autocovariances, which have only been obtained for the Gegenbauer process in (??) (see (??)). If there is more than one spectral pole/zero, only asymptotic behaviour has been established. Giraitis and Leipus (1995) showed that the autocovariances of (??) satisfy

$$
\gamma_{j} \sim K \sum_{k=0}^{h} j^{2 d_{k}-1} \cos \left(j \omega_{k}\right) \quad \text { as } j \rightarrow \infty .
$$

Thus $\pi_{j}, \psi_{j}$ and $\gamma_{j}$ have slow decay with oscillations depending on the different $\omega_{k}$. Eventually it is the largest persistence parameter which governs the behaviour of $\pi_{j}, \psi_{j}$ and $\gamma_{j}$.

The model (??) allows for spectral poles/zeros at any frequency $\omega_{j} \in[0, \pi]$. One particular case occurs when $\omega_{j}$ are seasonal frequencies, $\omega_{j}=2 \pi j / s, j=1,2, \ldots,[s / 2]$. Then (??) has been called "flexible ARFISMA" (Hassler (1994)) or "flexible (seasonal) ARMA $(p, d, q) s$ " (Ooms (1995)).

\section{ESTIMATION IN SCLM PROCESSES}

Hurst (1951) introduced the rescaled range statistic $(R / S)$ to measure long memory in the flows of the river Nile, and $R / S$ has been analysed, applied and extended by a number of subsequent authors. However $R / S$ does not extend readily to the SCLM context, so we explore alternative approaches.

Statistical inference in long memory processes can be parametric or semiparametric. Parametric methods are generally more efficient if they are based on a correct and complete specification of $f(\lambda)$, but even estimates of the persistence parameter, $d$ in (??), can be inconsistent if $f(\lambda)$ is misspecified at frequencies far from $\omega$. Semiparametric techniques, that only assume partial knowledge of $f(\lambda)$ around a known frequency (like in (??)) are less efficient but guarantee consistency under much more general circumstances. 


\subsection{Parametric Estimation}

Consider the covariance stationary process, $x_{t}$, satisfying

$$
\phi(L)\left(x_{t}-\mu_{0}\right)=\varepsilon_{t}
$$

where

$$
\phi(z)=1-\sum_{j=1}^{\infty} \phi_{j} z^{j} \quad, \quad \sum_{j=1}^{\infty} \phi_{j}^{2}<\infty
$$

$\mu_{0}=E x_{t}$ and the $\varepsilon_{t}$ have zero mean and are uncorrelated with variance $\sigma_{0}^{2}$, for all $t$. All the stationary and invertible processes described in previous sections can be written as (??) satisfying (??). Suppose that the $\phi_{j}$ and $\sigma_{0}^{2}$, as well as $\mu_{0}$, are unknown, but we know a function

$$
\phi(z ; \theta)=1-\sum_{j=1}^{\infty} \phi_{j}(\theta) z^{j}
$$

where $\theta$ is a $k \times 1$ vector such that there exists an unknown $\theta_{0}$ for which $\phi_{j}\left(\theta_{0}\right)=\phi_{j}$ for all $j$, and therefore $\phi\left(z ; \theta_{0}\right)=\phi(z)$. The spectral density of $x_{t}$ is given by

$$
f(\lambda)=\frac{\sigma_{0}^{2}}{2 \pi}\left|\phi\left(e^{i \lambda}\right)\right|^{-2}, \quad-\pi<\lambda \leq \pi
$$

and the lag- $j$ autocovariance by

$$
\gamma_{j}=\int_{-\pi}^{\pi} f(\lambda) \cos (j \lambda) \mathrm{d} \lambda
$$

For any admissible $\theta$ we introduce

$$
\begin{aligned}
\gamma_{j}(\theta) & =\frac{1}{2 \pi} \int_{-\pi}^{\pi} h(\lambda ; \theta) \cos (j \lambda) \mathrm{d} \lambda, \\
h(z ; \theta) & =\left|\phi\left(e^{i z} ; \theta\right)\right|^{-2} .
\end{aligned}
$$

In this section we consider so-called Gaussian estimates, although Gaussianity is not required to achieve good asymptotic properties. Denote by $\Delta(\theta)$ the $n \times n$ Toeplitz matrix with $(i, j)$ th element $\gamma_{i-j}(\theta)$, by 1 the $n \times 1$ vector of ones and by $x$ the $n \times 1$ vector of observations $\left(x_{1}, x_{2}, \ldots, x_{n}\right)^{\prime}$. For

$$
L_{a}\left(\theta, \mu, \sigma^{2}\right)=\frac{1}{2} \log \sigma^{2}+\frac{1}{2} \log |\Delta(\theta)|+\frac{1}{2 \sigma^{2}}(x-\mu \mathbf{1})^{\prime} \Delta(\theta)^{-1}(x-\mu \mathbf{1})
$$

define

$$
\left(\hat{\theta}_{a}, \hat{\mu}_{a}, \hat{\sigma}_{a}^{2}\right)=\arg \min _{\theta, \mu, \sigma^{2}} L_{a}\left(\theta, \mu, \sigma^{2}\right)
$$

where the minimization is carried out over an appropriate set. In case the $\varepsilon_{t}$ in (??) (and therefore $x_{t}$ ) are Gaussian, $\hat{\theta}_{a}$ is a maximum likelihood estimate of $\theta_{0}$.

As in other optimization problems introduced below, $\sigma_{0}^{2}$ and $\mu_{0}$ can be estimated in closed form and the nonlinear optimization carried out only with respect to $\theta$. Under regularity conditions $\hat{\theta}_{a}$ is consistent and

$$
\sqrt{n}\left(\hat{\theta}_{a}-\theta_{0}\right) \stackrel{d}{\rightarrow} N_{k}\left(0, \Omega^{-1}\right)
$$


where $\stackrel{d}{\rightarrow}$ means convergence in distribution, $N_{k}(\cdot, \cdot)$ is a $k$-variate normal and

$$
\Omega=\frac{1}{4 \pi} \int_{-\pi}^{\pi} \frac{\partial}{\partial \theta} \log h\left(\lambda ; \theta_{0}\right) \frac{\partial}{\partial \theta^{\prime}} \log h\left(\lambda ; \theta_{0}\right) \mathrm{d} \lambda .
$$

Since the function $h(z ; \theta)$ is known, $\Omega$ can be consistently estimated by, for example, substituting $\theta_{0}$ in (??) by a consistent estimate of it (e.g. $\hat{\theta}_{a}$ ). These asymptotic properties do not rely on $x_{t}$ being Gaussian, though under Gaussianity $\hat{\theta}_{a}$ is also asymptotically efficient.

We can approximate $L_{a}\left(\theta, \mu, \sigma^{2}\right)$ by

$$
L_{b}\left(\theta, \mu, \sigma^{2}\right)=\frac{1}{2} \log \sigma^{2}+\frac{1}{2 \sigma^{2}} \sum_{t=1}^{n} \varepsilon_{t}^{2}(\theta, \mu)
$$

where $\varepsilon_{t}(\theta, \mu)=\phi(L ; \theta)\left(x_{t}-\mu\right)$ and $x_{t}=0$ for $t \leq 0$. We call

$$
\left(\hat{\theta}_{b}, \hat{\mu}_{b}, \hat{\sigma}_{b}^{2}\right)=\arg \min _{\theta, \mu, \sigma^{2}} L_{b}\left(\theta, \mu, \sigma^{2}\right)
$$

a (nonlinear) least squares estimate. Under regularity conditions, $\hat{\theta}_{b}$ has the same asymptotic properties as $\hat{\theta}_{a}$.

Next define the centered periodogram

$$
I_{n}(\lambda ; \mu)=\frac{1}{2 \pi n}\left|\sum_{t=1}^{n}\left(x_{t}-\mu\right) e^{i t \lambda}\right|^{2} .
$$

Whittle (1953) proposed to approximate $L_{a}\left(\theta, \mu, \sigma^{2}\right)$ by

$$
L_{c}\left(\theta, \mu, \sigma^{2}\right)=\frac{1}{2 \pi} \int_{-\pi}^{\pi}\left\{\log \sigma^{2} h(\lambda ; \theta)+\frac{I_{n}(\lambda ; \mu)}{\sigma^{2} h(\lambda ; \theta)}\right\} \mathrm{d} \lambda .
$$

and the estimates

$$
\left(\hat{\theta}_{c}, \hat{\mu}_{c}, \hat{\sigma}_{c}^{2}\right)=\arg \min _{\theta, \mu, \sigma^{2}} L_{c}\left(\theta, \mu, \sigma^{2}\right) .
$$

Under regularity conditions, $\hat{\theta}_{c}$ has the same asymptotic properties as $\hat{\theta}_{a}$ and $\hat{\theta}_{b}$.

Finally define the (uncentered) periodogram

$$
I_{n}(\lambda)=\frac{1}{2 \pi n}\left|\sum_{t=1}^{n} x_{t} e^{i t \lambda}\right|^{2}
$$

Define the Fourier or harmonic frequencies $\lambda_{j}=2 \pi j / n$, and the discrete approximation to $L_{c}\left(\theta, \mu, \sigma^{2}\right)$ (see Hannan (1973b))

$$
L_{d}\left(\theta, \sigma^{2}\right)=\frac{1}{n} \sum_{j}^{\prime}\left\{\log \sigma^{2} h\left(\lambda_{j} ; \theta\right)+\frac{I_{n}\left(\lambda_{j}\right)}{\sigma^{2} h\left(\lambda_{j} ; \theta\right)}\right\}
$$

where $\sum_{j}^{\prime}$ runs over all $j=1, \ldots, n-1$, such that $0<h\left(\lambda_{j} ; \theta\right)<\infty$ for all admissible $\theta$. By omitting $j=0$ and $n$ we avoid the need to estimate $\mu_{0}$. Let

$$
\left(\hat{\theta}_{d}, \hat{\sigma}_{d}^{2}\right)=\arg \min _{\theta, \sigma^{2}} L_{d}\left(\theta, \sigma^{2}\right)
$$


where the minimization is over a compact subset of $R^{k+1}$. Then $\hat{\theta}_{d}$ typically has the same asymptotic properties as $\hat{\theta}_{a}, \hat{\theta}_{b}$ and $\hat{\theta}_{c}$ described above.

The relative computational needs of $\hat{\theta}_{a}, \hat{\theta}_{b}, \hat{\theta}_{c}$ and $\hat{\theta}_{d}$, which we call Gaussian estimates, depend on the parameterization we impose. In general, $\hat{\theta}_{b}$ is more easily calculated than $\hat{\theta}_{a}$ since it avoids the matrix inversion in (??) and $\hat{\theta}_{d}$ more easily calculated than $\hat{\theta}_{b}$ and $\hat{\theta}_{a}$ because $h(\lambda ; \theta)$ is typically of simpler form than $\varepsilon_{t}(\theta, \mu)$ and $\gamma_{j}(\theta)$. Moreover $\hat{\theta}_{d}$ makes especially convenient use of the fast Fourier transform.

The above discussion has made no reference to long memory or SCLM models, and in fact $\hat{\theta}_{a}, \hat{\theta}_{b}, \hat{\theta}_{c}$ and $\hat{\theta}_{d}$ and their asymptotic properties were originally obtained for short memory time series models such as stationary and invertible ARMA's (see for example Whittle (1953) or Hannan (1973b)). However the discussion also seems relevant to SCLM models. In fact, for long memory models with a spectral pole/zero only at the origin, Fox and Taqqu (1986), Dahlhaus (1989), Giraitis and Surgailis (1990), Heyde and Gay (1993) and Hosoya (1997) provide asymptotic properties for $\hat{\theta}_{c}$ which are identical to those earlier obtained for short memory processes (see e.g. (??)). Li and McLeod (1986) and Sowell $(1986,1992)$ discuss computational aspects of $\hat{\theta}_{a}$ for fractional ARIMA processes

$$
\Phi(L)(1-L)^{d}\left(x_{t}-\mu_{0}\right)=\Theta(L) \varepsilon_{t}
$$

where the zeros of $\Phi(z)$ and $\Theta(z)$ lie outside the unit circle. $\hat{\theta}_{b}$ for invertible, possibly nonstationary fractional ARIMA processes has been analysed by Beran (1995). Beran (1994b) proposed a modified version of $\hat{\theta}_{b}$ for long memory processes that is robust against outliers. Asymptotic theory for $\hat{\theta}_{d}$ has not been considered explicitly for long memory models with a spectral pole at zero frequency but it can be done by avoiding the spectral singularity with the omission of frequencies close to the origin in $L_{d}\left(\theta, \sigma^{2}\right)$. In case of long memory at frequency zero $\hat{\theta}_{d}$ has an extra advantage over $\hat{\theta}_{a}, \hat{\theta}_{b}$ and $\hat{\theta}_{c}$, because these are affected by $\hat{\mu}_{a}, \hat{\mu}_{b}$ and $\hat{\mu}_{c}$ which converge more slowly than $\sqrt{n}$ (see Vitale (1973), Adenstedt (1974) and Samarov and Taqqu (1988)), as discussed by Cheung and Diebold (1994) via Monte Carlo analysis.

The discussion of Gaussian estimates also seems relevant to SCLM models with spectral poles/zeros at known frequencies different from zero. Consider

$$
\Phi(L) \prod_{j=0}^{h}\left(1-2 L \cos \omega_{j}+L^{2}\right)^{d_{j}}\left(x_{t}-\mu_{0}\right)=\Theta(L) \varepsilon_{t}
$$

where $d_{j}>0$ for all $j$, and $d_{j}<1 / 2$ if $\omega_{j} \neq 0, \pi$, and $d_{j}<1 / 4$ if $\omega_{j}=0, \pi, \Theta(z)$ and $\Phi(z)$ have their roots outside the unit circle and the $\varepsilon_{t}$ are as before. In this case

$$
h(\lambda ; \theta)=\left|\frac{\Theta\left(e^{i \lambda}\right)}{\Phi\left(e^{i \lambda}\right)}\right|^{2} \prod_{j=0}^{h}\left(2\left(\cos \lambda-\cos \omega_{j}\right)\right)^{-2 d_{j}}
$$

where $\theta=\left(\Phi_{1}, \ldots, \Phi_{p}, \Theta_{1}, \ldots, \Theta_{q}, d_{0}, \ldots, d_{h}\right)^{\prime}$. Giraitis and Leipus (1995) obtain consistency of $\hat{\theta}_{c}$ but they do not establish the asymptotic distribution, although a non-Gaussian limit distribution 
is conjectured. For vector $x_{t}$ in (??) Hosoya $(1996,1997)$ considered a multivariate extension of $L_{c}\left(\theta, \mu, \sigma^{2}\right)$ and obtained an analogous result to (??), (??).

Following Kashyap and Eom (1988) we can also proceed by regressing $\log I_{n}\left(\lambda_{j}\right)$ on $\log h\left(\lambda_{j} ; \theta\right)$ over $j=1, \ldots, n-1$, though this approach leads to less efficient than Gaussian estimates. In fact Ray (1993) used this technique to estimate $d_{3}$ and $d_{12}$ in the SCLM process

$$
\phi_{0}(L) \phi_{3}\left(L^{3}\right) \phi_{12}\left(L^{12}\right)\left(1-L^{3}\right)^{d_{3}}\left(1-L^{12}\right)^{d_{12}} x_{t}=\theta_{0}(L) \theta_{3}\left(L^{3}\right) \theta_{12}\left(L^{12}\right) \varepsilon_{t}
$$

where the $\varepsilon_{t}$ are white noise. Ray (1993) used these estimates as a first step in the estimation of the complete model (??) for monthly IBM revenues.

\subsection{Semiparametric Estimation}

When we are interested only in estimation of the persistence parameter, $d$ in (??), we only need to specify $f(\lambda)$ around $\omega$ in order to obtain consistent estimates that we call semiparametric. This is a clear advantage with respect to parametric estimates that need a complete and correct specification of $f(\lambda)$ over the whole band of Nyqvist frequencies for consistency, though in the event of such specification the parametric estimates have the competing advantage of converging faster.

Due to their simplicity, perhaps the most popular semiparametric procedures are variants of the log-periodogram estimate introduced by Geweke and Porter-Hudak (1983). Consider a least squares regression of $\log I_{n}\left(\omega+\lambda_{j}\right)$ on $-2 \log \lambda_{j}$ and an intercept, where $I_{n}(\lambda)$ is the periodogram defined in (??) and $\lambda_{j}=2 \pi j / n$ are Fourier frequencies. The regression is carried out for $j=1, \ldots, m$, where the "bandwidth" $m$ is an integer between 1 and $n / 2$ and in practice is much less than $n$, and for asymptotic theory satisfies at least

$$
\frac{1}{m}+\frac{m}{n} \rightarrow 0 \quad \text { as } n \rightarrow \infty
$$

The original version, due to Geweke and Porter-Hudak (1983), uses instead of $-2 \log \lambda_{j}$ the regressor $-\log \left\{4 \sin ^{2}\left(\lambda_{j} / 2\right)\right\}$, but as indicated by Robinson (1995a), use of the simpler $-2 \log \lambda_{j}$, which corresponds more naturally to (??), leads to equivalent asymptotic properties. These authors assumed $\omega=0$, when, because $I_{n}(\lambda)$ is an even function, regression of $\log I_{n}\left(\lambda_{j}\right)$ on $-2 \log \left|\lambda_{j}\right|$ for $j= \pm 1, . ., \pm m$ is equivalent to using frequencies for $j=1, \ldots, m$. When $\omega \neq 0, \pi$, $I_{n}(\omega+\lambda)$ is not necessary symmetric about $\omega$ and information on both sides of the pole/zero can make a substantial difference. Thus a log-periodogram estimate for such $\omega$ is

$$
\hat{d}=-\frac{1}{2} \frac{\sum_{j= \pm 1}^{ \pm m} v_{j} \log I_{n}\left(\omega+\lambda_{j}\right)}{\sum_{j= \pm 1}^{ \pm m} v_{j}^{2}}
$$

where $v_{j}=\log |j|-\frac{1}{m} \sum_{1}^{m} \log l$. Work on estimating (??) with $\omega=0$ suggests two possible modifications to this scheme. Due to anomalous behaviour of the periodogram very close to a spectral pole/zero (see Robinson (1995a), Kunsch (1986) and Hurvich and Beltrao (1993,1994)), Kunsch (1986) and Robinson (1995a) trimmed out some frequencies close to $\omega$. The second 
type of modification is an efficiency improvement suggested by Robinson (1995a) and based on pooling adjacent periodogram ordinates. Incorporating these two suggestions we have the estimate

$$
\hat{d}^{(J)}=-\frac{1}{4} \frac{\sum_{k}^{\prime} v_{k}\left[\log \hat{I}_{\omega J k}+\log \tilde{I}_{\omega J k}\right]}{\sum_{k}^{\prime} v_{k}^{2}}
$$

where $\hat{I}_{\omega J k}=\sum_{j=1}^{J} I_{n}\left(\omega+\lambda_{k+j-J}\right), \tilde{I}_{\omega J k}=\sum_{j=1}^{J} I_{n}\left(\omega-\lambda_{k+j-J}\right), J$ is a positive integer (the pooling number) and $\sum_{k}^{\prime}$ is a sum over $k=l+J, l+2 J, \ldots, m$. When the pooling number, $J=1$, and the trimming number, $l=0$, then (??) reduces to (??). When $\omega=0$ Robinson (1995a) proved that under Gaussianity

$$
\sqrt{m}\left(\hat{d}^{(J)}-d\right) \stackrel{d}{\rightarrow} N\left(0, \frac{J \psi^{\prime}(J)}{4}\right) \quad \text { as } n \rightarrow \infty
$$

where $\psi^{\prime}(z)=\frac{\mathrm{d}}{\mathrm{d} z} \psi(z)$ and $\psi(z)$ is the digamma function defined as $\frac{\mathrm{d}}{\mathrm{d} z} \log \Gamma(z)$ where $\Gamma(z)$ is the Gamma function. The same asymptotics follow for $\omega \neq 0$ in (??) (see Arteche (1998)). For $\omega=0$ Velasco (1997c) relaxes the assumption of Gaussianity and only imposes boundness of the fourth moments of the $\varepsilon_{t}$ in (??) to obtain consistency and asymptotic normality (using a suitably tapered periodogram) with variance $3 J \psi^{\prime}(J) / 4$. Note that tapering increases the variance. Still for $\omega=0$, and assuming Gaussianity, Velasco (1997a) proves consistency of $\hat{d}^{(J)}$ for the non-stationary case $d \in[1 / 2,1)$ and also shows that $\hat{d}^{(J)}$ is asymptotically normal with variance $J \psi^{\prime}(J) / 4$ for the non-tapered estimate if $d \in[1 / 2,3 / 4)$, and $3 J \psi^{\prime}(J) / 4$ for $d \in[1 / 2,3 / 2)$ in the tapered case. The good properties in finite samples of $\hat{d}^{(1)}$ for $d \in[1 / 2,1)$ are shown in Hurvich and Ray (1995). These results seem to extend straightforwardly to the case $\omega \neq 0$.

Related with the parametric Gaussian estimates described in the previous section, Kunsch (1987) and Robinson (1995b) considered a semiparametric approximation of $L_{d}\left(\theta, \sigma^{2}\right)$ in (??). The estimate, $\tilde{d}$, is the argument that minimizes

$$
Q(C, d)=\frac{1}{2 m} \sum_{j= \pm 1}^{ \pm m}\left\{\log C\left|\lambda_{j}\right|^{-2 d}+\frac{\left|\lambda_{j}\right|^{2 d}}{C} I_{n}\left(\omega+\lambda_{j}\right)\right\}
$$

where $m$ satisfies at least (??). The estimate $\tilde{d}$ has been called the Gaussian semiparametric or local Whittle estimate. When $\omega=0$ only frequencies on one side of $\omega$ are used, due to the symmetry of $I_{n}(\lambda)$ at the origin. Without requiring Gaussianity, Robinson (1995b) obtained consistency and asymptotic normality for the case $\omega=0$ such that

$$
\sqrt{m}(\tilde{d}-d) \stackrel{d}{\rightarrow} N(0,1 / 4)
$$

Note that $\tilde{d}$ is asymptotically more efficient than $\hat{d}^{(J)}$ because $J \psi^{\prime}(J) \downarrow 1$ as $J \rightarrow \infty$. The same asymptotics hold for $\omega \neq 0$ (see Arteche (1998)). Velasco (1997b) extended Robinson's results to non-stationary processes obtaining consistency for $d \in[1 / 2,1)$ and asymptotic normality when $d \in[1 / 2,2 / 3)(d \in[1 / 2,3 / 4)$ under Gaussianity). A multivariate extension of this estimator is studied by Lobato (1995). 
Robinson (1994b) proposed an alternative technique to estimate $d$ in case

$$
f(\omega+\lambda) \sim L\left(\frac{1}{|\lambda|}\right)|\lambda|^{-2 d} \quad \text { as } \lambda \rightarrow 0
$$

where $L(z)$ is a slowly varying function, that is a positive measurable function satisfying

$$
\frac{L(t z)}{L(z)} \rightarrow 1 \quad \text { as } z \rightarrow \infty \quad \text { for all } t>0 .
$$

Note that (??) specializes to (??) when $L(z)$ is a constant. The proposed "averaged periodogram" estimate is

$$
\hat{d}_{q m \omega}=\frac{1}{2}-\frac{\log \left\{\hat{F}\left(q \lambda_{m}\right) / \hat{F}\left(\lambda_{m}\right)\right\}}{2 \log q}
$$

where

$$
\hat{F}(\lambda)=\frac{2 \pi}{n} \sum_{j= \pm 1}^{ \pm[\lambda n / 2 \pi]} I_{n}\left(\omega+\lambda_{j}\right),
$$

and $q \in(0,1)$ is a user chosen number and $m$ again satisfies at least (??). With only second moment restrictions and without requiring Gaussianity, Robinson (1994b) showed the consistency of $\hat{d}_{q m \omega}$ for $\omega=0$. Assuming Gaussianity, Lobato and Robinson (1996a) obtained the asymptotic distribution of $\hat{d}_{q m \omega}$ for $\omega=0$. This is normal for $d \in(0,1 / 4)$ and non-normal (related to Rossenblatt processes) for $d \in(1 / 4,1 / 2)$. The same properties are likely to hold for $\omega \neq 0$.

Janacek (1982) introduced an alternative method to estimate $d$ through estimation of the Fourier coefficients of $\log f(\lambda)$ using the log-periodogram. Although originally this estimate was proposed for long memory at frequency zero, Janacek claimed that this method can be naturally extended to SCLM time series.

A number of other semiparametric estimates have been proposed for the $\omega=0$ case that seem capable of extending to general $\omega$, such as the time domain ones of Robinson (1994c), and the one of Parzen (1986) and Hidalgo and Yajima (1996) that achieves an efficiency improvement over the estimates described above.

\section{TESTING SEASONAL/CYCLICAL INTEGRATION AND COINTEGRATION}

The characteristics of the process generating the series depend strongly on the value of the persistence parameter, $d$. In particular, $d$ determines if the process has long memory (stationary or non-stationary), short memory or negative memory (invertible or non-invertible). Some interesting situations that may require a rigorous test are

a) $d=0$ (short memory) against $d>0$ (long memory) or $d<0$ (negative memory),

b) $d=1 / 2$ ("just" non-stationarity) against $d>1 / 2$ (non-stationarity) or $d<1 / 2$ (stationarity),

c) $d=-1 / 2$ ("just" non-invertibility) against $d>-1 / 2$ (invertibility) or $d<-1 / 2$ (noninvertibility). 
The hypotheses involved in a) can be tested using simple $t$ tests based on the estimates and their asymptotic distributions described in Section 4 or by Lagrange Multiplier tests as those proposed in the parametric case by Robinson (1994a) or in a semiparametric setting by Lobato and Robinson (1996b). $t$-tests on b) and c) can be carried out using those estimates whose asymptotic properties hold for non-stationary or non-invertible processes.

Traditionally, interest has focused on testing the possibility of unit roots where $d$ in (??) is an integer. Some early work is due to Dickey, Hasza and Fuller (1984) who test the possibility of a seasonal unit root of the form

$$
\left(1-L^{s}\right) x_{t}=\varepsilon_{t} \quad t=1,2, \ldots
$$

where the $\varepsilon_{t}$ are iid $\left(0, \sigma^{2}\right)$ random variables, against the alternative

$$
x_{t}=\alpha x_{t-s}+\varepsilon_{t}
$$

with $|\alpha|<1$. They provide percentiles for the proposed test statistic. One of the limitations of this procedure is that it is a joint test for unit roots at the origin and seasonal frequencies, $\omega_{h}=2 \pi h / s, h=1,2, \ldots,[s / 2]$ (see (??) for the case $s=4$ ). Furthermore the alternative is a specified form of $s$-th order autoregressive process. Hylleberg et al. (1990), using quarterly data, extended this procedure allowing for an individual test at zero and at every seasonal frequency that is robust to behaviour at other frequencies. Some extensions of this procedure to monthly data are Beaulieu and Miron (1993) and Franses (1991). The null hypothesis in each case is pure integrability $\left(I_{\omega}(1)\right)$ and the alternative is pure stationarity or short memory $\left(I_{\omega}(0)\right)$. Canova and Hansen (1995) extended the test of Kwiatkowsky et al. (1992) to the seasonal case, testing the null of stationarity $\left(I_{\omega}(0)\right)$ against the alternative of pure integration $\left(I_{\omega}(1)\right)$. Bearing in mind the properties of these two types of test, that basically differ in the specification of the null and alternative, the simultaneous use of both procedures has been advised in order to test for pure integrability. The same conclusion of both types of test (that is one rejects and the other does not reject the null) provides strong evidence in favour of the result implied by both procedures. If one test contradicts the other, then we need a more thorough analysis. In this case we may have fractional integration.

A general test, based on the parametric model (??) and allowing for fractional and integer $I_{\omega}(d)$ as null and alternative, has been proposed by Robinson (1994a) and applied to quarterly macroeconomic data by Gil-Alaña and Robinson (1997). Suppose

$$
\begin{array}{rlc}
\phi(L)\left(x_{t}-\mu\right) & =u_{t} & t=1,2, \ldots \\
x_{t} & =0 & t \leq 0
\end{array}
$$

where $u_{t}$ is a short memory covariance stationary sequence with zero mean, and $\phi(z)$ is a known function. Consider the function $\phi(z ; \vartheta)$ where $\vartheta$ is a $p$-dimensional vector of real valued parameters such that $\phi(z ; \vartheta)=\phi(z)$ if and only if

$$
H_{0}: \vartheta=0
$$


The hypotheses of principle interest entail $\phi$ of the form

$$
\phi(L ; \vartheta)=(1-L)^{d_{0}+\vartheta_{i_{0}}}\left\{\prod_{j=1}^{h-1}\left(1-2 L \cos \omega_{j}+L^{2}\right)^{d_{j}+\vartheta_{i_{j}}}\right\}(1+L)^{d_{h}+\vartheta_{i_{h}}}
$$

where for each $j, \vartheta_{i_{j}}=\vartheta_{l}$ for some $l$ and for each $l$ there is at least one $j$ such that $\vartheta_{i_{j}}=\vartheta_{l}$. The null hypothesis is that the $p \times 1$ vector $(p \leq h+1) \vartheta=\left(\vartheta_{1}, \vartheta_{2}, \ldots, \vartheta_{p}\right)^{\prime}$ is a vector of zeros. Thus fractional seasonal and cyclical integration is allowed in the null and alternative in contrast with the focus on testing for a unit root against autoregressive alternatives in much of the literature. To avoid estimation of the persistence parameters, Robinson (1994a) used a score test although undoubtedly the same asymptotic behaviour can be expected of Wald and likelihood ratio tests. When $u_{t}$ is white noise the proposed test statistic is

$$
R=\frac{n}{\tilde{\sigma}^{4}} \tilde{a}^{\prime} \tilde{A}^{-1} \tilde{a}
$$

where $\tilde{\sigma}^{2}=\frac{1}{n} \sum_{1}^{n} u_{t}^{2}, u_{t}=\phi(L ; 0) x_{t}, \tilde{a}=-\frac{2 \pi}{n} \sum_{j}^{\prime} \Psi\left(\lambda_{j}\right) I_{u}\left(\lambda_{j}\right), I_{u}(\lambda)$ is the periodogram of $u_{t}$ defined in (??), $\Psi(\lambda)=\operatorname{Re}\left\{\frac{\partial}{\partial \vartheta} \log \phi\left(e^{i \lambda} ; 0\right)\right\}$ and $\tilde{A}=\frac{2}{n} \sum_{j}^{\prime} \Psi\left(\lambda_{j}\right) \Psi\left(\lambda_{j}\right)^{\prime}$ where the primed sum is over $\lambda_{j} \in M=\left\{\lambda:-\pi<\lambda<\pi, \lambda \notin\left(\omega_{l}-\lambda_{1}, \omega_{l}+\lambda_{1}\right), l=0,1, \ldots, h\right\}$ and $\omega_{l}$ are the distinct poles of $\Psi(\lambda)$ on $(-\pi, \pi]$. Asymptotically equivalent expressions for $\tilde{a}$ and $\tilde{A}$ can be found in Robinson (1994a), as well as a time domain test statistic. Robinson (1994a) also proposed a modification of $R$ that allows for parametric weak correlation in $u_{t}$ so long as its spectrum is bounded and bounded away from zero and of known parametric form. Unlike the techniques earlier described these procedures have the advantage of being standard in the sense that the test statistic has a $\chi_{p}^{2}$ limit distribution under the null and a limiting non-central $\chi_{p}^{2}$ distribution against Pitman or local alternatives, and are asymptotically locally most powerful.

Hylleberg et al. (1990) considered the possibility of seasonal cointegration, which they defined as

A pair of series each of which are integrated at frequency $\omega$ are said to be cointegrated at that frequency if a linear combination of the series is not integrated at $\omega$.

Hylleberg et al. (1990) pointed out that in case of several spectral poles (as for example $x_{t}$ in (??)) the procedure in Engle and Granger (1987) to test for cointegration at zero frequency is invalid, so that prior to any test for cointegration we have to filter the data in such a way that only the pole at the frequency where we suspect the cointegration occurs remains. For instance, if we want to test for cointegration at the origin, we have first to remove seasonal roots, for example by applying the seasonal summation operator, $S(L)=\left(1+L+\ldots+L^{s-1}\right)$, to the original series and then performing a standard cointegration test such as those discussed in Engle and Granger (1987).

Engle and Granger (1987) and Hylleberg et al. (1990) consider only the possibility that a linear combination of $I_{\omega}(1)$ processes is $I_{\omega}(0)$. But our definition of SCLM or $I_{\omega}(d)$ processes allows for the possibility of fractional integration and cointegration. In this sense Engle et al. (1989) define cyclical cointegration in the following manner, 
A vector of series $x_{t}$, each component $I_{\omega}(d)$ (integrated of order $d$ at frequency $\omega$ ), may be said to be cointegrated at that frequency if there exists a vector $\alpha_{\omega}$ such that $z_{t}^{\omega}=\alpha_{\omega}^{\prime} x_{t}$ is integrated of lower order at $\omega$.

As in the definition of SCLM, the case of cointegration at every seasonal frequency is known as seasonal cointegration.

\section{ESTIMATION OF THE FREQUENCY $\omega$}

Most analyses of SCLM models assume that the frequency $\omega$ where the spectral pole occurs is known. Of course, seasonal frequencies are known, but in cyclical time series, $\omega$ may well be unknown.

The literature on estimating $\omega$ in cyclical long memory is of recent date and it is of interest to consider first earlier work on estimating frequency in an alternative model, namely the deterministic periodic time series

$$
x_{t}=\alpha_{0} \sin \omega t+\beta_{0} \cos \omega t+u_{t}
$$

where $u_{t}$ is stationary with mean zero and spectral density, $f_{u}(\lambda)$, continuous and positive at $\omega$. Whittle (1952) found that the least squares estimate of $\omega$ in (??), $\hat{\omega}$, is the periodogram maximizer and has a variance $O\left(n^{-3}\right)$. Walker (1971) (for $u_{t}$ white noise) and Hannan (1971, 1973a) extended Whittle's work and, without assuming Gaussianity, found that for $\omega \neq 0, \pi$,

$$
n^{3 / 2}(\hat{\omega}-\omega) \stackrel{d}{\rightarrow} N\left(0, \frac{48 \pi f_{u}(\omega)}{\alpha_{0}^{2}+\beta_{0}^{2}}\right) .
$$

In case $\omega=0, \pi$, Hannan (1973a) showed that there exists an integer valued random variable, $n_{0}$, with $P\left(n_{0}<\infty\right)=1$ such that $\hat{\omega}=\omega$ for $n>n_{0}$, so that $\hat{\omega}$ will be equal to the value it estimates for a large enough sample size. Mackisack and Poskitt (1989) proposed a different technique based on the maximization of the transfer function calculated by fitting high order autoregressions to $x_{t}$. Only $\sqrt{n}$-consistency for $\omega \in(0, \pi)$ is rigorously proved (although it is claimed that the variance of the estimate is $O\left(n^{-\frac{5}{2}}\right)$ when the order of the autoregression is $O\left(n^{\frac{1}{2}}\right)$ ), and their method is computationally intensive. A different approach has been suggested by Quinn and Fernandes (1991). The technique is based on fitting $\operatorname{ARMA}(2,2)$ models in an iterative way and they propose a simple algorithm that converges rapidly. The same asymptotic distribution, (??), as the maximizer of the periodogram is obtained. A similar procedure with the same asymptotic distribution is described in Truong-Van (1990).

In (??) only one sinusoidal component is assumed. However a multiple finite number of components can describe seasonal or cyclical movement,

$$
x_{t}=\sum_{j=1}^{r}\left\{\alpha_{j} \cos \left(\omega_{j} t\right)+\beta_{j} \sin \left(\omega_{j} t\right)\right\}+u_{t} .
$$


In this context estimation of $r$, the number of cosinusoids, has been treated by Quinn (1989), Kavalieris and Hannan (1994), Hannan (1993) and Wang (1993) among others. Estimation of the $\omega_{j}$ has been analysed in Chen (1988a,b), Walker (1971) and Kavalieris and Hannan (1994).

The estimation of $\omega$ in cyclical long memory models may be necessary to determine the periodicity of the cycle and as a first step prior to estimation of remaining parameters. Yajima (1995) considered the model

$$
f(\lambda ; \omega, \theta)=g(\lambda ; \omega, \theta)|\lambda-\omega|^{-2 d} \quad \omega \in[0, \pi] \text { and } 0<d<1 / 2, d \in \theta,
$$

where $\theta$ is a parameter vector including $d$, and the function $g$ obeys some regularity conditions, such that the GARMA process is a special case of (??). The estimate of $\omega$ considered by Yajima is the periodogram maximizer. He obtains $n^{\alpha}$-consistency under Gaussianity for any $\alpha \in(0,1)$ and shows that the Whittle estimates of $\theta$ obtained by minimizing

$$
U_{n}(\hat{\omega}, \theta)=\int_{-\pi}^{\pi}\left\{\log f(\lambda ; \hat{\omega}, \theta)+\frac{I_{n}(\lambda)}{2 \pi f(\lambda ; \hat{\omega}, \theta)}\right\} \mathrm{d} \lambda
$$

are $\sqrt{n}$-consistent and asymptotically normal. Yajima does not provide any distribution theory for his estimate of $\omega$, but a non-normal distribution is conjectured.

Chung (1996a,b) obtained an estimate of $\eta=\cos \omega$ in Gegenbauer processes,

$$
\phi(L)\left(1-2 L \eta+L^{2}\right)^{d}\left(x_{t}-\mu\right)=\theta(l) \varepsilon_{t}
$$

and claimed asymptotic properties for conditional sum of squares estimates (cf. (??)) including a limit non-normal distribution for the estimate of $\omega$ but a normal limit distribution for the estimates of the remaining parameters.

A joint estimation of all the frequencies $\omega_{j}, j=0,1, \ldots, h$, and the rest of long and short memory parameters in the model (??) is proposed by Giraitis and Leipus (1995). They obtain consistency of the Whittle estimates obtained minimizing $U_{n}(\omega, \theta)$ defined in (??), but no asymptotic distribution.

Hidalgo (1997) proposes an alternative semiparametric technique to estimate $\omega$ in a process satisfying (??) with $d \in(0,1 / 2)$. The estimate $\hat{\omega}_{H}$ is the argument that maximizes the estimate of $d$ proposed by Hidalgo and Yajima (1997),

$$
\hat{d}^{*}=\frac{1}{m} \sum_{p=1}^{m} \hat{d}_{p},
$$

where $\hat{d}_{p}=a_{1} / a_{2}$ and

$$
\begin{aligned}
& a_{1}=\frac{1}{p} \sum_{l=1}^{p} w(l) \log \hat{f}_{p}\left(\lambda_{l}\right)-\left(\frac{1}{p} \sum_{l=1}^{p} w(l)\right) \log \hat{f}_{p}\left(\lambda_{p+1}\right), \\
& a_{2}=-2 \int_{0}^{1} w(u) \log u \mathrm{~d} u
\end{aligned}
$$

where $w(l)=(l / p)^{\frac{1}{c}}-(l / p)^{\frac{1}{c+1}}, c>1$ and $\hat{f}_{p}\left(\lambda_{l}\right)$ is a particular moving average of periodogram ordinates at frequencies close to $\omega$. Without assuming Gaussianity Hidalgo (1997) shows that $n k^{-\frac{1}{2}}\left(\hat{\omega}_{H}-\omega\right)$, for $k \rightarrow \infty$ suitably slowly with $n$, has a normal limit distribution. 


\section{CONCLUSION AND EXTENSIONS}

This paper has discussed modelling and inference in SCLM processes having spectral density satisfying (??). The combination of seasonal or cyclic behaviour and long memory can lead to several extensions:

1. The autoregressive coefficients $\pi_{j}$ in (??) of the SCLM model in (??) can be useful for forecasting. Although obtaining $C_{k}^{(\hat{d})}$ from a given estimate of $d, \hat{d}$, can be done recursively, the accurate generation of the $\pi_{j}$ 's gets more difficult as the number of spectral poles and the sample size increase, and deserves attention.

2. The definition (??) of SCLM imposes an asymptotically symmetric behaviour in $f(\lambda)$ around $\omega$. Nevertheless, if $\omega \neq 0, f$ need not actually be symmetric. We can generalize from SCLM to SCALM (Seasonal Cyclical Asymmetric Long Memory) processes by defining

$$
\begin{aligned}
& f(\omega+\lambda) \sim C_{1} \lambda^{-2 d_{1}} \\
& f(\omega-\lambda) \sim C_{2} \lambda^{-2 d_{2}} \quad \text { as } \quad \lambda \rightarrow 0^{+}
\end{aligned}
$$

where $C_{1}, d_{1}$ can be different from $C_{2}, d_{2}$. Then (??) is a restriction of (??) that happens when $C_{1}=C_{2}=C$ and $d_{1}=d_{2}=d$. Discussion of (??) has began in Arteche (1998) and Arteche and Robinson (1998).

3. Some financial series such as asset returns appear to be approximately serially uncorrelated. However, there are nonlinear transformations, such as squares, that can exhibit autocorrelation as modelled in the extensive $\mathrm{ARCH}$ and stochastic volatility literature, following Engle (1982) or Taylor $(1986,1994)$. Moreover there is evidence of long memory autocorrelation in the squares of some series. The first model that causes this effect is the general GARCH process proposed by Robinson (1991), who uses it as an alternative in testing for no-ARCH. His model is sufficiently general to describe SCLM behaviour in the squares, as may be appropriate in financial data. 


\section{References}

[1] ADENSTEDT, R.K. (1974). On Large Sample Estimations for the Mean of a Stationary Random Sequence. Annals of Statistics, Vol.2, No.6, 1095-1107.

[2] ANDEL, J. (1986). Long-Memory Time Series Models. Kybernetica, Vol. 22, 105-123.

[3] ARTECHE, J. (1998). Seasonal and Cyclical Long Memory in Time Series. Ph.D. Thesis, LSE, University of London.

[4] ARTECHE, J. and ROBINSON, P.M. (1998). Semiparametric Inference in Seasonal and Cyclical Long Memory Processes. Preprint.

[5] BEAULIEU, J.J. and MIRON, J.A. (1993). Seasonal Unit Roots in Aggregated U.S. Data. Journal of Econometrics, 55, 305-328.

[6] BELL, W.R. and HILLMER, S.C. (1984). Issues Involved with the Seasonal Adjustment of Economic Time Series. Journal of Business and Economic Statistics, 2, 291-320.

[7] BERAN, J. (1994a). Statistics for Long-Memory Processes. Monographs on Statistics and Applied Probability 61. Chapman and Hall.

[8] BERAN, J. (1994b). On a Class of M-Estimators for Gaussian Long-Memory Models. Biometrika, 81, 4, 755-766.

[9] BERAN, J. (1995). Maximum Likelihood Estimation of the Differencing Parameter for Invertible Short and Long Memory Autoregressive Integrated Moving Average Models. $J$. R. Statist. Soc. B, Vol. 57, No. 4, 659-672.

[10] BOX, G.E.P. and JENKINS, M. (1976). Time Series Analysis: Forecasting and Control. Holden-Day, San Francisco.

[11] CANOVA, F. and HANSEN, B.E. (1995). Are Seasonal Patterns Constant over Time? A Test for Seasonal Stability. Journal of Business and Economic Statistics, Vol. 13, No. 3, $237-252$

[12] CARLIN, J.B. and DEMPSTER, A.P. (1989). Sensitivity Analysis of Seasonal Adjustments: Empirical Case Studies. Journal of the American Statistical Association, 84: 6-20.

[13] CHAN, N.H. and TERRIN, N. (1995). Inference for Unstable Long-Memory Processes with Applications to Fractional Unit Root Autoregressions. The Annals of Statistics. Vol. 23, No. 5, 1662-1683.

[14] CHAN, N.H. and WEI, C.Z. (1988). Limiting Distributions of Least Squares Estimates of Unstable Autoregressive Processes. The Annals of Statistics. Vol. 16, N0. 1, 367-401. 
[15] CHEN, Z.G. (1988a). An Alternative Consistent Procedure for Detecting Hidden Frequencies. Journal of Time Series Analysis, Vol. 9, No. 3, 301-317.

[16] CHEN, Z.G. (1988b). Consistent Estimate for Hidden Frequencies in a Linear Process. Advances in Probability, 20, 295-314.

[17] CHEUNG, Y.W. and DIEBOLD, F.X. (1994). On Maximum Likelihood Estimation of the Differencing Parameter of Fractionally Integrated Noise with Unknown Mean. Journal of Econometrics 62, 301-316.

[18] CHUNG, C.F. (1996a). Estimating a Generalized Long-Memory Process. Journal of Econometrics 73, 237-259.

[19] CHUNG, C.F. (1996b). A Generalized Fractionally Integrated Autoregressive MovingAverage Process. Journal of Time Series Analysis, Vol.17, No. 2, 111-140.

[20] DAGUM, E.B. (1980). The X-11 ARIMA Seasonal Adjustment Method. Statistics Canada, Catalogue No. 12-564E.

[21] DAHLHAUS,R. (1989). Efficient Parameter Estimation for Self-Similar Processes. The Annals of Statistics, 17: 1749-1766.

[22] DICKEY, D.A., HASZA, D.P. and FULLER, W.A. (1984). Testing for Unit Roots in Seasonal Time Series. Journal of the American Statistical Association 79, 355-367.

[23] ENGLE, R.F. (1982). Autoregressive Conditional Heteroscedasticity with Estimates of the Variance of United Kingdom Inflation. Econometrica, Vol. 50, No. 4, 987-1007.

[24] ENGLE, R.F. and GRANGER, W.J. (1987). Co-integration and Error Correction: Representation, Estimation, and Testing. Econometrica, Vol. 55, No. 2, 251-276.

[25] ENGLE, R.F., GRANGER, C.W.J. and HALLMAN, J.J. (1989). Merging Short and LongRun Forecasts: An Application of Seasonal Cointegration to Monthly Electricity Sales Forecasting. Journal of Econometrics 40, 45-62.

[26] FOX, R. and TAQQU, M.S. (1986). Large Sample Properties of Parameter Estimates for Strongly Dependent Stationary Gaussian Time Series. The Annals of Statistics, 14: 517-532.

[27] FRANSES, P.H. (1991). Model Selection and Seasonality in Time Series. Tinbergen Institute Series, No. 18.

[28] FRANSES, P.H. and OOMS, M. (1995). A Periodic Long-Memory $\operatorname{ARFIMA}\left(0, D_{s}, 0\right)$ Model for Quarterly UK Inflation. Report 9511/A, Erasmus University Rotterdam, The Netherlands.

[29] GEWEKE, J. and PORTER-HUDAK, S. (1983). The Estimation and Application of LongMemory Time Series Models. Journal of Time Series Analysis, 4: 221-238. 
[30] GIL-ALAÑA, L.A. and ROBINSON, P.M. (1997). Testing of Unit Root and Other Nonstationary Hypothesis in Macroeconomic Time Series. Journal of Econometrics, forthcoming.

[31] GIRAITIS, L. and LEIPUS, R. (1995). A Generalized Fractionally Differencing Approach in Long-Memory Modelling. Liet. Matem. Rink. 35, No.1, 65-81.

[32] GIRAITIS, L. and SURGAILIS, D. (1990). A Central Limit Theorem for Quadratic Forms in Strongly Dependent Linear Variables and its Application to Asymptotic Normality of Whittle's Estimate. Probability Theory and Related Fields, 86: 87-104.

[33] GRAY, H.L., ZHANG, N.F. and WOODWARD, W.A. (1989). On Generalized Fractional Processes. Journal of Time Series Analysis, 10: 233-257.

[34] GRAY, H.L., ZHANG, N.F. and WOODWARD, W.A. (1994). On Generalized Fractional Processes- A Correction. Journal of Time Series Analysis, Vol.15, No.5, 561-562.

[35] HANNAN, E.J. (1963). The Estimation of Seasonal Variation in Economic Time Series. Journal of the American Statistical Association, 58, 31-44.

[36] HANNAN, E.J. (1964). The Estimation of a Changing Seasonal Pattern. Journal of the American Statistical Association, 59, 1063-1077.

[37] HANNAN, E.J. (1967). Measurement of a Wandering Signal Amid Noise. Journal of Applied Probability, 4, 90-102.

[38] HANNAN, E.J. (1971). Non-linear Time Series Regression. Journal of Applied Probability, 8, 767-780.

[39] HANNAN, E.J. (1973a). The Estimation of Frequency. Journal of Applied Probability, 10, $510-519$.

[40] HANNAN, E.J. (1973b). The Asymptotic Theory of Linear Time Series Models. Journal of Applied Probability, 10, 130-145.

[41] HANNAN, E.J. (1993). Determining the Number of Jumps in a Spectrum. Developments in Time Series Analysis. Ed. Subba Rao. London: Chapman \& Hall, 127-138.

[42] HANNAN, E.J., TERREL, R.D. and TUCKWELL, N.E. (1970). The Seasonal Adjustment of Economic Time Series. International Economic Review, Vol. 2, No. 1, 24-52.

[43] HARVEY, A.C. (1989). Forecasting Structural Time Series Models and the Kalman Filter. Cambridge University Press.

[44] HASSLER, U. (1994). (Mis)specification of Long-Memory in Seasonal Time Series. Journal of Time Series Analysis, 15: 19-30. 
[45] HEYDE, C.C. and GAY, R. (1993). Smoothed Periodogram Asymptotics and Estimation for Processes and Fields with Possible Long-Range Dependence. Stochastic Processes and their Applications, 45, 169-182.

[46] HIDALGO, J. (1997). Estimation of the Pole of Long-Range Processes. Preprint.

[47] HIDALGO, J. and YAJIMA, Y. (1997). Semiparametric Estimation of the Long-Range Parameter. Preprint

[48] HOSKING, J.R.M. (1981). Fractional Differencing. Biometrika, 68: 165-176.

[49] HOSKING, J.R.M. (1984) Modelling persistence in hydrological time series using fractional differencing. Water Resour. Res. 20, 1898-1908.

[50] HOSOYA, Y. (1996). The Quasi-Likelihood Approach to Statistical Inference on Multiple Time Series with Long-Range Dependence. Journal of Econometrics, 73, 217-236.

[51] HOSOYA, Y. (1997). A Limit Theory for Long-Range Dependence and Statistical Inference in Related Models. Annals of Statistics, 25, 105-137.

[52] HURST, H.E. (1951). Long-Term Storage Capacity of Reservoirs. Transaction of the American Society of Civil Engineers 1, 519-543.

[53] HURVICH, C.M. and BELTRAO, K.I. (1993). Asymptotics for the Low-Frequency Ordinates of the Periodogram of Long-Memory Time Series. Journal of Time Series Analysis, Vol.14, No.5, 455-472.

[54] HURVICH, C.M. and BELTRAO, K.I. (1994). Acknowledgment of Priority for "Asymptotics for the Low-Frequency Ordinates of the Periodogram of Long-Memory Time Series". Journal of Time Series Analysis, 15, 64.

[55] HURVICH, C.M. and RAY, B.K. (1995). Estimation of the Memory Parameter for Nonstationary or Noninvertible Fractionally Integrated Processes. Journal of Time Series Analysis, Vol.16, No.1, 17-42.

[56] HYLlEBERG, S., ENGLE, R.F., GRANGER, C.W.J. and YOO, B.S. (1990). Seasonal Integration and Cointegration. Journal of Econometrics 44, 215-238.

[57] JONAS, A.J. (1983). Persistent Memory Random Processes, PhD Thesis, Department of Statistics, Harvard University.

[58] KASHYAP, R.L. and EOM, K.B. (1988). Estimation in Long-Memory Time Series Model. Journal of Time Series Analysis, Vol.9, No.1, 35-41.

[59] KAVALIERIS, L. and HANNAN, E.J. (1994). Determining the Number of Terms in a Trigonometric Regression. Journal of Time Series Analysis, Vol.15, No.6, 613-625. 
[60] KUNSCH, H.R. (1986). Discrimination between Monotonic Trends and Long-Range Dependence. Journal of Applied Probability, 23, 1025-1030.

[61] KUNSCH, H.R. (1987). Statistical Aspects of Self-similar Processes. Proceedings of the First World Congress of the Bernoulli Society, (Yu. Prohorov and V.V. Sazanov, eds.) 1, 67-74, VNU Science Press, Utrecht.

[62] KWIATKOWSKY, D., PHILliPS, P.C.B., SCHMIDT, P. and SHIN, Y. (1992). Testing the Null Hypothesis of Stationarity Against the Alternative of a Unit Root. Journal of Econometrics, 54, 159-178.

[63] LI, W.K. and McLEOD, A.I. (1986). Fractional Time Series Modelling. Biometrika 73, 217-221.

[64] LOBATO, I.N. (1995). Multivariate Analysis of Long-Memory Time Series in the Frequency Domain. Ph.D. thesis, University of London.

[65] LOBATO, I.N. and ROBINSON, P.M. (1996a). Averaged Periodogram Estimate of LongMemory. Journal of Econometrics, 73, 303-324.

[66] LOBATO, I.N. and ROBINSON, P.M. (1996b). A Nonparamemtric Test for I(0). Forthcoming, Review of Economic Studies.

[67] MACKISACK, M.S. and POSKITT, D.S. (1989). Autoregressive Frequency Estimation. Biometrika, 76, 3, 565-575.

[68] MANDELBROT, B.B and VAN NESS, J.N. (1968). Fractional Brownian Motions, Fractional Noises and Applications. SIAM review 10, 422-437.

[69] NERLOVE,M. (1964). Spectral Analysis of Seasonal Adjustment Procedures. Econometrica,Vol.32, No.3, 241-286.

[70] OOMS, M. (1995). Flexible Seasonal Long-Memory and Economic Time Series. Preprint.

[71] OSBORN,D.R. (1991). The Implications of Periodically Varying Coefficients for Seasonal Time Series Processes. Journal of Econometrics, 48: 373-384.

[72] PARZEN, E. (1986). Quantile Spectral Analysis and Long-Memory Time Series. Journal of Applied Probability, 23A, 41-54.

[73] PORTER-HUDAK, S. (1990). An Application of Seasonal Fractionally Differenced Model to the Monetary Aggregates. Journal of the American Statistical Association, Vol.85, No.410, 338-344.

[74] QUINN, B.G. (1989). Estimating the Number of Terms in a Sinusoidal Regression. Journal of Time Series Analysis, Vol.10, No.1, 71-75. 
[75] QUINN, B.G. and FERNANDES, J.M. (1991). A Fast Efficient Technique for the Estimation of Frequency. Biometrika, 78, 3, 489-97.

[76] RAY, B.K. (1993). Long-Range Forecasting of IBM Product Revenues Using a Seasonal Fractionally Differenced ARMA Model. International Journal of Forecasting 9, 255-269.

[77] ROBINSON, P.M. (1991). Testing for Strong Serial Correlation and Dynamic Conditional Heteroskedasticity in Multiple Regression. Journal of Econometrics, 47, 67-84.

[78] ROBINSON, P.M. (1994a). Efficient Tests of Non-Stationary Hypothesis. Journal of the American Statistical Association, 89: 1420-1437.

[79] ROBINSON, P.M. (1994b). Semiparametric Analysis of Long-Memory Time Series. The Annals of Statistics, Vol.22, 515-539.

[80] ROBInSON, P.M. (1994c). Time Series with Strong Dependence. Advances in Econometrics, Cambridge University Press, Vol.1, 47-95.

[81] ROBINSON, P.M. (1995a). Log-Periodogram Regression of Time Series with Long-Range Dependence. The Annals of Statistics, Vol.23, No.3, 1048-1072.

[82] ROBINSON, P.M. (1995b). Gaussian Semiparametric Estimation of Long-Range Dependence. The Annals of Statistics, Vol.23, No.5, 1630-1661.

[83] SAMAROV, A. and TAQQU,M. (1988). On the Efficiency of the Sample Mean in Long Memory Noise. Journal of Time Series Analysis, Vol.9, No.2, 191-200.

[84] SHISKIN, J., YOUNG, A.H. and MUSGRAVE, J.C. (1967). The X-11 Variant of the Census Method II Seasonal Adjustment Program. Technical Paper no. 15, Washington DC: Bureau of the Census, US Department of Commerce.

[85] SOWELL, F. (1986). Fractionally Integrated Vector Time Series. Ph.D. dissertation, Duke University, Durham, N.C.

[86] SOWELL, F. (1992). Maximum Likelihood Estimation of Stationary Univariate Fractionally Integrated Time Series Models. Journal of Econometrics 53, 105-188.

[87] TAYLOR, S.J. (1986). Modelling Financial Time Series. Wiley \& Sons.

[88] TAYLOR, S.J. (1994). Modelling Stochastic Volatility: A Review and Comparative Study. Mathematical Finance, Vol.4, No.2, 183-204.

[89] TIAO, G.C. and GRUPE, M.R. (1980). Hidden Periodic Autoregressive-Moving Average Models in Time Series Data. Biometrika, 67, 2, 365-373.

[90] Troutman, B.M. (1979). Some Results in Periodic Autoregression. Biometrika, 66, 2, 219-228. 
[91] TRUONG-VAN, B. (1990). A New Approach to Frequency Analysis with Amplified Harmonics. Journal of the Royal Statistical Society B, 52, No.1, 203-221.

[92] VELASCO, C. (1997a). Non-Stationary Log-Periodogram Regression. Preprint.

[93] VELASCO, C. (1997b). Gaussian Semiparametric Estimation of Non-stationary Time Series. Preprint.

[94] VELASCO, C. (1997c). Non-Gaussian Log-Periodogram Regression. Preprint.

[95] VITALE, R.A. (1973). An Asymptotically Efficient Estimate in Time Series Analysis. Quarterly Applied Mathematics, XXX, 421-440.

[96] WALKER, A.M. (1971). On the Estimation of a Harmonic Component in a Time Series with Stationary Independent Residuals. Biometrika, 58, 1, 21-36.

[97] WANG, X. (1993). An AIC Type Estimator for the Number of Cosinusoids. Journal of Time Series Analysis, 14, 431-440.

[98] WHITTLE, P. (1952). The Simultaneous Estimation of a Time Series Harmonic Components and Covariance Structure. Trabajos de Estadística, 3, 43-57.

[99] WHITTLE, P. (1953). Estimation and Information in Stationary Time Series. Ark. Mat., 2: $423-434$.

[100] YAJIMA, Y. (1995). Estimation of the Frequency of Unbounded Spectral Densities. Preprint.

[101] YONG, C.H. (1974). Asymptotic Behaviour of Trigonometric Series. Chinese Univ. Hong Kong. 